BJP $161-12 / 5 / 2006-182653$

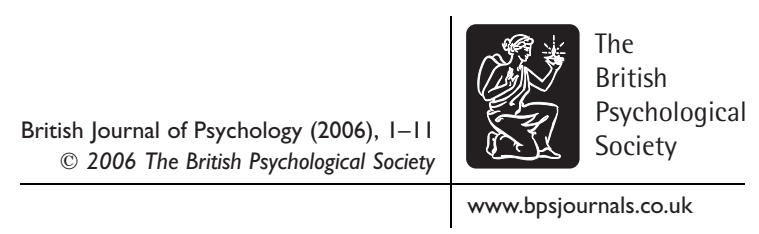

\title{
Of two minds: Sceptic-proponent collaboration within parapsychology
}

\author{
Marilyn Schlitz', Richard Wiseman ${ }^{2 *}$, Caroline Watt ${ }^{3}$ \\ and Dean Radin' \\ 'Institute of Noetic Sciences, USA \\ ${ }^{2}$ University of Hertfordshire, UK \\ ${ }^{3}$ University of Edinburgh, UK
}

\begin{abstract}
The first author, a proponent of psychic ability, and the second, a sceptic, have been conducting a systematic programme of collaborative sceptic-proponent research in parapsychology. This has involved carrying out joint experiments in which each investigator individually attempted to mentally influence the electrodermal activity of participants at a distant location. The first two collaborations obtained evidence of 'experimenter effects', that is, experiments conducted by the proponent obtained significant results but those conducted by the sceptic did not. This paper describes a new collaborative study that attempted to replicate our previous findings and explore potential explanations for past results. The new study failed to replicate our previous findings. The paper investigates whether the results obtained in our initial studies may have been caused by a genuine psychic effect. This third experiment failed to replicate this finding because some aspect of the study disrupted the production of that effect, or whether the results from our first two studies represented chance findings or undetected subtle artifacts, and the results obtained in the present study accurately reflect the absence of a remote detection of staring effect. The implications of this work are discussed, along with the benefits of conducting collaborative work for resolving disagreements in other controversial areas of psychology.
\end{abstract}

For well over a hundred years scientists have explored the possible existence of psychic ability (Edge, Morris, Palmer, \& Rush, 1986). This work has generated considerable controversy, with proponents arguing that the cumulative research supports the existence of such abilities (see, e.g. Bem \& Honorton, 1994; Utts, 1991) and sceptics claiming that the evidence suffers from possible methodological or statistical problems (see, e.g. Alcock, 2003; Hyman, 1994). Researchers on both sides of the debate have tended to collaborate with colleagues who share similar beliefs about the existence of psychic ability. This is unfortunate, as joint sceptic-proponent collaborations offer the

* Correspondence should be addressed to Richard Wiseman, University of Hertfordshire, Hatfield Campus, College Lane, Hatfield, Herts ALIO 9AB, UK (e-mail: r.wiseman@herts.ac.uk). 
BJP $161-12 / 5 / 2006--182653$

potential to help resolve key areas of disagreement (e.g. Hyman \& Honorton, 1986). For several years, the second author (a sceptic about the existence of psychic ability) has collaborated with the first author (a proponent of such abilities) on a systematic programme of joint sceptic-proponent experimental work within parapsychology (Wiseman \& Schlitz, 1997, 1999). This research has involved jointly conducted experiments exploring the possible existence of a commonly reported phenomenon, the 'sense of being stared at.' This paper describes our latest joint study.

Surveys suggest that 70 to $90 \%$ of the population has experienced an uneasy feeling of being stared at, only to turn around and discover somebody looking at them (Coover, 1913; Braud, Shafer, \& Andrews, 1993a). Research into this phenomenon has a long and distinguished history, with initial papers on the topic being published around the turn of the last century by two pioneers of modern day psychology: E.B. Titchener (1898) and J.E. Coover (1913). The first experimental investigation into the phenomenon was conducted by Coover at Stanford University. It involved an experimenter sitting behind participants, either staring directly at their backs or looking away and then asking them to decide whether they had just been stared at. Subsequent work has involved increasingly sophisticated methodological and statistical procedures. For example, researchers have minimized potential experimenter-participant sensory cues by employing one-way mirrors (Peterson, 1978) and closed-circuit television systems (Braud et al., 1993a; Braud, Shafer, \& Andrews, 1993b), and created a more sensitive dependent measure of participant's arousal by recording their electrodermal activity (EDA), rather than asking them to report whether they are being stared at (Braud et al., 1993a, 1993b).

The basic experimental procedure that has evolved involves the participant and experimenter being located in two separate, sensory-isolated rooms. A closed-circuit television system feeds a live image of the participant to a monitor in the experimenter's room and, at randomly determined times, the experimenter either stares at this image with the intention of physiologically arousing the participant ('stare' trials) or looks away from the monitor and disengages his/her intention ('no-stare' trials). The participant's EDA is continuously recorded during the experiment and any significant differential effects observed in EDA between 'stare' and 'no-stare' trials is inferred to reflect the existence of psychic functioning. A recent meta-analytic review of 15 experiments using these types of procedures revealed a small, but statistically significant, overall effect (Schmidt, Schneider, Utts, \& Walach, 2004).

The first author (MS) has conducted numerous parapsychological studies that have obtained positive results and has argued in favour of certain types of psychic ability (e.g. Schlitz, 2001; Schlitz \& Honorton, 1992). In contrast, the second author (RW) has carried out several studies that have obtained chance results and has published critiques about the adequacy of experimental findings in supporting the existence of psychic abilities (e.g. Milton \& Wiseman, 1999; Wiseman \& Greening, 2002). In the early 1990s each of us carried out separate studies into the remote detection of staring. The experiments conducted by RW showed no evidence of psychic functioning (Wiseman \& Smith, 1994; Wiseman, Smith, Freedman, Wasserman, \& Hurst, 1995) whilst MS's study yielded significant results (Schlitz \& LaBerge, 1994).

Such 'experimenter effects' are common within parapsychology. Many researchers have argued that it is vitally important to establish why they occur, both in terms of assessing past parapsychological research and attempting to replicate studies in the future (e.g. Palmer, 1989a, 1989b, 1997; Smith, 2003). Indeed, the importance of understanding this issue is such that Palmer (1986) noted: 
Q1

'...the experimenter effect is the most important challenge facing modern parapsychology. It may be that we will not be able to make too much progress in other areas of the field until the puzzle of the experimenter effect is solved'.

The experimenter effects obtained in the remote staring studies individually conducted by MS and RW in the early 1990 s are open to several competing interpretations. For example, MS's study may have contained an experimental artifact absent from RW's procedure. Alternatively, MS may have worked with more psychically gifted participants than RW or been more skilled at eliciting participants' psi ability. It is also possible that MS and RW created desired results via their own psi abilities or fraud. In 1996, the authors agreed to carry out some joint studies in the hope of determining whether such effects would continue to be obtained and, if this were the case, exploring the mechanisms behind such findings.

The first joint project involved conducting two studies in RW's laboratory, using the same psychophysiology equipment, the same experimental procedures and drawing participants from the same pool ( $N=16$ in each study). The only difference between the studies was that one involved MS as experimenter/starer, whilst the other involved RW carrying out these roles. The two studies obtained different results. The EDA of MS's participants was significantly higher in stare than in no-stare trials (effect size [es] $=.50 ;{ }^{1} p=.04$, two-tailed), whereas the EDA of RW's participants showed no such effect (es $=.11 ; p=.64$, two-tailed).

The second joint project (Wiseman \& Schlitz, 1999) took place at MS's laboratory and again involved two studies employing the same procedures, equipment and participant pool ( $N=35$ for each study). Once again, MS's experiment showed a small but statistically significant effect (es =.33; $p=.05$, two-tailed), whereas RW's did not (es $=.07 ; p=.69$, two-tailed).

The present study attempted to replicate our previous findings and evaluate two hypotheses that may explain the pattern of results obtained in these studies. In our previous work the experimenter both interacted with the participant at the start of each session (explaining the purpose of the study, administering questionnaires, etc) and carried out the staring/no-staring. The differing outcomes might thus have been due to either different ways in which the experimenters interacted with participants or how they performed their staring. The present study employed a $2 \times 2$ cross-over design to evaluate these hypotheses (see Diagram 1). In two of the conditions (A \& D), either RW or MS met the participant at the start of the session (i.e. acted as 'greeter') and then the same person carried out the stare/no-stare trials (i.e. acted as 'sender'). In the other two conditions (B \& C), either RW or MS acted as greeter and the other acted as sender. If the previous experimental results were due to the way in which MS interacted with participants, one would predict a main effect of greeter. If, however, they were due to the way in which the stare/no-stare trials were conducted, one would expect a main effect of sender.

The notion that MS's success may be due to the positive rapport that she generates with participants was also assessed by correlating the effect size in each of the sessions in which she acted as greeter with her level of rapport with the participant concerned. A measure of greeter-participant rapport was obtained by videotaping each session and having the resulting tapes coded by two independent raters. The hypothesis that MS's

'ES calculated from zI $\sqrt{ } N$ using a z-score obtained from a Wilcoxon signed rank test (see Wiseman \& Schlitz, 1997). 
BJP $161-12 / 5 / 2006--182653$

Diagram I. Study design

\begin{tabular}{llc}
\hline & MS sender & RW sender \\
\hline MS greeter & A & B \\
RW greeter & C & D \\
\hline
\end{tabular}

success might be due to the way in which she carries out the stare and no-stare trials was assessed by correlating effect sizes from each of the sessions in which she acted as sender, with a questionnaire measure of her expectation of success and degree of focus that was completed immediately after the sending period.

\section{Participants}

The 100 participants who took part in the study (32 male, 68 female; mean age: 49, age range: 21-86) were either staff members of The Institute of Noetic Sciences or local volunteers and were recruited through word-of-mouth, posters and e-mail postings. ${ }^{2}$

\section{Layout of rooms and apparatus}

The experiment took place in two rooms of the Institute of Noetic Sciences' psychophysiology laboratory. The larger room contained a Lindgren/ETS double steelwalled, electromagnetically and acoustically shielded chamber and two computers used to record EDA and control the random assignment of stare and no-stare trials. The shielded chamber contained equipment for measuring participants' EDA (see below), a reclining chair and a video-camera. A smaller room, 15 metres away from the shielded room and behind a double wall, contained a desk, chair and a television monitor linked to the video-camera in the shielded room. The acoustic shielding between the chamber and the remote observation room attenuated sounds by approximately $100 \mathrm{~dB}$, making inadvertent auditory communications between participants and the experimenter highly unlikely. Visual communication between the experimenter and participants was prevented because the shielded chamber had solid steel walls with no windows, its door remained closed throughout the stare/no-stare trials and it was located in a different room behind a double wall and two doors.

The measurement and recording of participants' EDA followed guidelines recommended by Schmidt and Walach (2000). The participant sat in a comfortable chair and both the humidity and temperature in the shielded chamber were monitored. EDA measurements in the form of skin conductance level (SCL) were obtained through two $9 \mathrm{~mm}$ (diameter) silver/silver chloride electrodes filled with an isotonic paste, placed on the participant's non-dominant palm with double-sided adhesive collars and connected to an EDA amplifier on a Biopac M150 system with a constant voltage $(0.5 \mathrm{v})$ in the direct-coupled mode. These signals were recorded at a rate of 25 samples per second. The physiological and video signals were routed outside the chamber through fibre optics to maintain the integrity of the electromagnetic and acoustic shielding.

\footnotetext{
${ }^{2}$ This study was originally intended to involve 140 participants with 35 participants in each of the four cells. However, due to unforeseen constraints on time and resources, a decision was made in April 2004 to reduce the number of participants to 100 ( 25 in each cell). This decision was made prior to any examination of the data.
} 
BJP $161-12 / 5 / 2006--182653$

\section{Assignment of stare and no-stare trials}

Each session consisted of 20 stare and 20 no-stare trials. Each trial lasted 20 seconds and trials were separated from one another by a randomly determined inter-trial interval (ITI) of between 5 and 15 seconds. These random ITIs helped prevent participants from guessing when the next trial would begin and also precluded the possibility of their physiological rhythms accidentally synchronizing to the uniform length of the stare and no-stare trials (see Schlitz \& LaBerge, 1994). To help prevent artifacts due to participant relaxation or habituation over the course of the session, stare and no-stare trials were scheduled in groups of four, with each group consisting of either a 'stare, no-stare, no-stare, stare' (ABBA) or 'no-stare, stare, stare, no-stare' (BAAB) order (Wiseman \& Schlitz, 1997). The ten ABBA or BAAB orders used in each session were randomly assigned by a pseudo-random algorithm initiated just prior to each session. During stare trials the monitor in the remote observation room displayed a live image of the participant. At all other times the monitor displayed a live image of an empty reception room. A computer-controlled video switch automatically directed Q2 the appropriate video signal. A Microsoft Visual Basic program controlled all aspects of the experiment.

\section{Videotape coding and sender questionnaire}

A video-camera unobtrusively filmed the greeter and participant whilst the greeter described the experimental procedure, administered the consent form, asked the participant to complete various questionnaires and attached the electrodes for EDA recording. Following the completion of the study, these tapes were transferred to 12 different videotapes (with each tape containing 3-10 sessions ${ }^{3}$ ) and two independent raters coded the degree of greeter-participant rapport in each session in a random order (inter-rater reliability $=.65$ ). Both raters were unaware of the identity of the two experimenters and, when this identity was revealed after coding, they reported no prior knowledge of either MS or RW. ${ }^{4}$ An overall measure of rapport was created by averaging the two individuals' ratings for each session.

The Sender Questionnaire was completed by the sender immediately after the completion of the stare and no-stare trials and asked them to rate the degree to which they felt focused during the sending period and the degree to which they felt the session would demonstrate a remote staring effect on two scales that ranged from 0 (very low) to 100 (very high).

\section{Procedure}

Before the experiment, a research assistant randomly assigned equal numbers of participants to each of the four conditions and prepared two envelopes for each session. ${ }^{5}$ One envelope contained the name of the greeter and the other contained the name of the sender. Prior to the start of a session, RW and MS opened the 'greeter'

\footnotetext{
${ }^{3}$ There was no video recording for the first 13 sessions because $C W$ was not present to arrange filming and 7 other sessions did not record properly due to equipment malfunction. Therefore, the total number of sessions rated was 80 .

${ }^{4}$ See Watt, Schlitz, Wiseman, and Radin (2005) for a description of the other questionnaire measures and ratings employed in this study.

${ }^{5}$ Due to an administrative error discovered after the experiment had been completed, one of the four cells contained 24 participants and another contained 26.
} 
BJP $161-12 / 5 / 2006--182653$

6

Marilyn Schlitz et al.

envelope together. The designated greeter then met the participant, obtained informed consent, explained the nature of the experiment, showed them to the shielded chamber and connected the EDA electrodes to the Biopac equipment. Participants were aware that the study was concerned with the remote detection of staring and were asked to remain psychologically open to any such influence during the stare and no-stare trials. Participants were also aware that the individual who would act as the sender may or may not be the person who had acted as the greeter. After the participant was securely housed in the shielded chamber, both experimenters met in a distant office to open the 'sender' envelope. The designated experimenter then went to the remote observation room and carried out the stare and no-stare trials. During stare trials the experimenter quietly directed his/her attention towards the participant; during no-stare trials the experimenter directed his/her attention away from the participant. ${ }^{6}$ Participants were blind to the identity of the starer.

\section{Results}

In our previous collaborative projects the participant's mean SCL was used for the dependent measure. Prior to conducting the present study a more sensitive way of using participants' EDA was devised to obtain a measure of their autonomic arousal. First, the difference in each participant's mean SCL for the 20 stare and 20 no-stare trials was determined. Next, an exhaustive permutation analysis was used to determine the exact probability of the observed difference by comparing it to the outcomes obtained in all the other ways in which the order of the stare and no-stare trials could have been randomly assigned. Each resulting $p$ value was then transformed into a one-tailed $z$ score, such that a score of zero would indicate no difference in EDA between stare and no-stare trials, a positive $\mathrm{z}$ score would indicate greater EDA in the stare than no-stare condition and a negative $\mathrm{z}$ score would indicate greater EDA in the no-stare than stare condition. Based on the design of the present study, a 2 (RW or MS as greeter) $\times 2$ (RW or MS as sender) between-factors ANOVA was used to evaluate the resulting $\mathrm{z}$ scores. The main effects of greeter, $F(4,93)=0.46, p=.50$, and sender, $F(4,93)=0.21, p=.64$, and the interaction between these factors, $F(4,93)=0.04, p=.85$, failed to reach significance (see Table 1: All $p$ values are two-tailed).

The correlation between the individual session outcome and degree of greeterparticipant rapport during the sessions in which MS acted as greeter was not significant (Spearman's $r=-.028, p=.86$, two-tailed, $N=40$ ). The correlations between the session outcome and sender's self-rated level of focus and expectation of success in the sessions in which MS acted as sender were also non-significant (Focus: Spearman's $r=-.061, p=.69$, two-tailed, $N=46$; Expectation: $r=-.13, p=.40$, two-tailed, $N=46$ ). MS's overall degree of focus and expectation of success were not particularly high, being close to the mid-point of the rating scale (Focus: $M=51.2, S D=17.1$; Expectation: $M=52.7, S D=12.4$ ), suggesting that she was not especially confident of demonstrating some form of psychic functioning during the periods in which she acted as sender.

Post hoc analyses compared the results obtained in this present study with the findings from our earlier work. In our previous studies, Wilcoxon signed rank tests

\footnotetext{
${ }^{6}$ For additional informational about the psychological procedures used by RW and MS during the sending period, see Watt, Wiseman, and Schlitz (2002).
} 
Table I. Means and standard deviations (in parentheses) for sender and greeter conditions

\begin{tabular}{lrrr}
\hline & \multicolumn{1}{c}{ MS sender } & \multicolumn{1}{c}{ RW sender } & \multicolumn{1}{c}{ Totals } \\
\hline MS greeter & $0.012(0.84) N=25$ & $0.143($ I.04) $N=24$ & $0.076(0.94) N=49$ \\
RW greeter & $-0.087(I .16) N=25$ & $-0.032(0.95) N=26$ & $-0.059(1.05) N=51$ \\
Totals & $-0.037(I .00) N=50$ & $0.052(0.99) N=50$ & $0.007(0.99) N=100$ \\
\hline
\end{tabular}

revealed that MS's studies obtained significantly different mean EDA during stare versus no-stare trials but that this was not the case for RW's experiments. Identical analyses of the present data failed to reveal the same pattern. When MS acted as both greeter and sender (Condition A in Diagram 1), participants' EDA was not significantly different in stare versus no-stare trials $(N=25$; stare trials $M=1.24, S D=1.23$; no-stare trials $M=1.24, S D=1.21 ; \mathrm{z}$ score [corrected for ties] $=-.17 ; p$ [two-tailed] $=.87$, $\left.\mathrm{es}^{7}=-.03\right)$. This was also the case in condition $\mathrm{D}$, where RW acted as both greeter and sender $(N=26$; stare trials $M=2.65, S D=2.64$; no-stare trials $M=2.67, S D=2.24$; $\mathrm{z}$ score $[$ corrected for ties] $=-.35 ; p$ [two-tailed $]=.72$, es $=-.07$ )

\section{Discussion}

This study aimed to build upon our previous joint research by exploring whether the significant effects obtained in our previous work were due to the way in which MS interacted with participants towards the start of each session or how she carried out the stare and no-stare trials. Participants' ability to detect a remote stare was not influenced by whether MS acted as the greeter or sender. In addition, the study yielded nonsignificant correlations between the outcome of individual sessions and (i) greeterparticipant rapport ratings when MS acted as the greeter and (ii) MS's self-reported level of focus and positive expectation when she acted as sender. As such, the results of this study do not provide any evidence to suggest that these factors play a significant role in explaining our previous findings. In addition, results from the condition in which MS acted as both greeter and sender failed to yield any significant evidence of a remote staring effect and thus did not replicate our previous findings. As a result, the findings from our three joint experiments are open to two main competing interpretations.

First, MS's results in our two initial studies may have been caused by a genuine remote staring effect and this third experiment failed to replicate this finding because some aspect of the study disrupted the production of that effect. Although it is impossible to falsify this position, it is difficult to identify any obvious factors that might have prevented an alleged remote detection of staring effect from operating. MS was working with a group of participants who, for the most part, held a strong belief in the existence of psychic functioning and the study was conducted in a setting that was both familiar to her and supportive of the possibility of psychic ability. However, it is possible that a more subtle, unanticipated and uncontrolled factor may have disrupted the production of an overall effect. For example, the study took place in one- to two-week intensive blocks of testing over a two-year period and both experimenters informally reported experiencing a loss in motivation and interest as the experiment continued. To

${ }^{7} E S=$ Wilcoxon $z / \sqrt{ } N$. 
BJP $161-12 / 5 / 2006--182653$

8

Marilyn Schlitz et al.

examine whether this apparent loss of interest had a disruptive effect on experimental results, the outcome for individual sessions was correlated with the order in which the sessions were conducted. In fact, the outcome of the sessions conducted by RW or MS did not show any change over time when they acted as either the greeter (MS: $r=-.01$, $p=.92, N=49$; RW: $r=.07, p=.64, N=51$ ) or the sender (MS: $r=-.04, p=.80$, $N=50 ; \mathrm{RW}: r=.09, p=.55, N=50$ ).

Second, it is possible that the results from our first two studies represented chance findings or undetected subtle artifacts and that the results obtained in the present study accurately reflect the absence of a remote detection of staring effect. It is certainly the case that the methodology and statistical analyses employed in this third study were more sophisticated than the techniques and procedures used in our previous work. For example, the equipment used to measure and record participants' EDA was superior to that employed in our previous two studies (see Schmidt \& Walach, 2000) and the safeguards against sensory leakage during this latest experiment were far better than those used in our second study. However, such improvements were the result of a process of natural development that often takes place when researchers repeat their studies, rather than being driven by any concern that our previous findings were the result of any obvious artifacts.

Given that people tend to interpret ambiguous evidence in alignment with their prior beliefs (see, e.g. Roe, 1999), it is predicted that proponents of psychic ability will tend to favour the first interpretation of the data outlined above and sceptics the latter. However, the inconsistent nature of our findings does not allow for a firm acceptance or rejection of either interpretation and the issue will only be resolved by further research. The controversy generated by research into the possible existence of psychic abilities reflects the theoretical and practical importance of the questions raised by such potential abilities,and we believe this justifies the additional work needed to help resolve the type of inconsistent results reported here.

However, this series of experiments demonstrates that it is possible to conduct fruitful collaborative research involving both sceptics and proponents and it offers the potential of a more productive route than more traditional forms of sceptic-proponent debate (e.g. Honoton, 1985; Hyman, 1985). The joint project described here reduces the likelihood of perpetuating unconstructive rhetoric because sceptics and proponents are actively engaged in the same study and the procedures employed should minimize methodological flaws and maximize the procedures that proponents believe to be conducive to psychic functioning. In addition, opportunities for explaining away the results post hoc are limited since both parties are directly involved in the experiment and the interpretation of the data.

There are, however, several barriers that may hinder this type of collaborative venture. In many controversial areas of psychology, communities of researchers with opposing views tend not to attend the same conferences, publish in the same journals or even read the same type of academic articles and books (Blackmore, 1989). Additional barriers include an inherent distrust of one another fuelled by ideological differences, personal beliefs and past involvement in acrimonious debates. Our experience suggests that there is considerable value in trying to overcome these barriers and carry out systematic and collaborative ventures. It is hoped that the studies described here will encourage researchers working in other controversial areas (e.g. the role of trance in hypnosis, false memory syndrome, unorthodox forms of psychotherapy and complementary and alternative medicine) to engage in similar joint projects and that such work will help advance our understanding of the phenomena underlying these controversies. 


\section{Acknowledgements}

Our thanks to the Samueli Institute for Information Biology, the University of Hertfordshire, the Perrott-Warrick fund and the Institute of Noetic Sciences for supporting this work. Thanks also to Jenny Matthews for her invaluable assistance in conducting this research.

\section{References}

Alcock, J. (2003). Give the null hypothesis a chance: Reasons to remain doubtful about the existence of Psi. Journal of Consciousness Studies, 10(6-7), 29-50.

Bem, D. J., \& Honorton, C. (1994). Does Psi exist? Replicable evidence for an anomalous process of information transfer. Psychological Bulletin, 115(1), 4-18.

Blackmore, S. (1989). What do we really think: A survey of parapsychologists and sceptics. Journal of the Society for Psychical Research, 55(814), 251-262.

Braud, W., Shafer, D., \& Andrews, S. (1993a). Reactions to an unseen gaze (remote attention): A review, with new data on autonomic staring detection. Journal of Parapsychology, 57, 373-390.

Braud, W., Shafer, D., \& Andrews, S. (1993b). Further studies of autonomic detection of remote staring: Replications, new control procedures, and personality correlates. Journal of Parapsychology, 57, 391-409.

Coover, J. E. (1913). The feeling of being stared at. American Journal of Psychology, 24, 570-575.

Edge, H. L., Morris, R. L., Palmer, J., \& Rush, J. H. (1986). Foundations of parapsychology. London: Routledge \& Kegan Paul.

Honorton, C. (1985). Meta-analysis of Psi Ganzfeld research: A response to Hyman. Journal of Parapsychology, 49(1), 51-92.

Hyman, R. (1985). The ganzfeld psi experiment: A critical appraisal. Journal of Parapsychology, 49, 3-49.

Hyman, R. (1994). Anomaly or artifact? Comments on Bem and Honorton. Psychological Bulletin, $115,19-24$.

Hyman, R., \& Honorton, C. (1986). A joint communiqué: The psi ganzfeld controversy. Journal of Parapsychology, 50, 350-364.

Milton, J., \& Wiseman, R. (1999). Does Psi exist? Lack of replication of an anomalous process of information transfer. Psychological Bulletin, 125(4), 387-391.

Palmer, J. (1986). ESP research findings: The process approach. In H. L. Edge, R. L. Morris, J. Palmer \& J. H. Rush (Eds), Foundations of parapsychology (pp. 184-222). London: Routledge \& Kegan Paul.

Palmer, J. (1989a). Confronting the experimenter effect. Parapsychology Review, 20(4), 1-4.

Palmer, J. (1989b). Confronting the experimenter effect Part 2. Parapsychology Review, 20(5), $1-5$.

Palmer, J. (1997). The challenge of experimenter Psi. European Journal of Parapsychology, 13, 110-125.

Peterson, D. M (1978). Through the looking glass: An investigation of extra-sensory detection of being stared at. M.A. Thesis. University of Edinburgh.

Roe, C. A. (1999). Critical thinking and belief in the paranormal: A re-evaluation. British Journal of Psychology, 90(1), 85-98.

Schlitz, M. J. (2001). Boundless mind: Coming of age in parapsychology. Journal of Parapsychology, 65(4), 335-350.

Schlitz, M. J., \& Honorton, C. (1992). Ganzfeld psi performance within an artistically gifted population. Journal of the American Society for Psychical Research, 86, 83-98.

Schlitz, M. J., \& LaBerge, S. (1994). Autonomic detection of remote observation: Two conceptual replications. In D. J. Bierman (Ed.), Proceedings of the Parapsychological Association 37th Annual Convention, 352-360. 
BJP 161-12/5/2006--182653

\section{I0 Marilyn Schlitz et al.}

Schmidt, S., Schneider, R., Utts, J., \& Walach, H. (2004). Distant intentionality and the feeling of being stared at: Two meta-analyses. British Journal of Psychology, 95, 235-247.

Schmidt, S., \& Walach, H. (2000). Electrodermal activity (EDA): State-of-the-art measurement and techniques for parapsychological purposes. Journal of Parapsychology, 64, 139-164.

Smith, M. D. (2003). The role of the experimenter in parapsychological research. Journal of Consciousness Studies, 10, 69-84.

Titchener, E. B. (1898). The feeling of being stared at. Science, 8, 895-897.

Utts, J. (1991). Replication and meta-analysis in parapsychology. Statistical Science, 6(4), 363-403.

Watt, C., Schlitz, M., Wiseman, R., \& Radin, D. (2005). Experimenter differences in a remote staring study. Research brief accepted for presentation at the 2005 Parapsychological Association Convention.

Watt, C., Wiseman, R., \& Schlitz, M. (2002). Tacit information in remote staring research: The Wiseman-Schlitz interviews. Paranormal Review, 24, 18-25.

Wiseman, R., \& Greening, E. (2002). The mind machine: A mass participation experiment into the possible existence of extrasensory perception. British Journal of Psychology, 93, 487-499.

Wiseman, R., \& Schlitz, M. (1997). Experimenter effects and the remote detection of staring. Journal of Parapsychology, 61, 197-207.

Wiseman, R., \& Schlitz, M. (1999). Replication of experimenter effect and the remote detection of staring. Proceedings of the 42nd Annual Convention of the Parapsychological Association, 471-479.

Wiseman, R., \& Smith, M.D. (1994). A further look at the detection of unseen gaze. Proceedings of the 37th Annual Convention of the Parapsychological Association, 465-478.

Wiseman, R., Smith, MD., Freedman, D., Wasserman, T., \& Hurst, C. (1995). Two further experiments concerning the remote detection of an unseen gaze. Proceedings of the 38th Annual Convention of the Parapsychological Association, 480-490.

Received 15 December 2004; revised version received 28 August 2005 
BJP $161-12 / 5 / 2006-182653$

Of two minds

II

\section{Author Queries}

JOB NUMBER: 161

JOURNAL: BJP

Q1 Quote from Palmer (1986). Please provide page number for quote.

Q2 Please check whether this Microsoft Visual Basic Program is a registered trade mark? 